\title{
Respiratory Syncytial Virus Outbreak in the Basic Military Training Camp of the Republic of Korea Air Force
}

\author{
Won-Ju Park ${ }^{1,2}$, Seok-Ju Yoo ${ }^{2,3}$, Suk-Ho Lee ${ }^{2}$, Jae-Woo Chung ${ }^{4}$, Keun-Ho Jang ${ }^{1}$, Jai-Dong Moon ${ }^{1}$ \\ ${ }^{1}$ Department of Occupational and Environmental Medicine, Chonnam National University Hwasun Hospital, Hwasun; ${ }^{2}$ Aerospace Medical Re- \\ search Center, Republic of Korea Air Force, Cheongwon; ${ }^{3}$ Department of Preventive Medicine, Dongguk University College of Medicine, Gyeongju; \\ ${ }^{4}$ Department of Laboratory Medicine, Dongguk University Ilsan Hospital, Goyang, Korea
}

Objectives: An outbreak of acute febrile illness occurred in the Republic of Korea Air Force boot camp from May to July 2011. An epidemiological investigation of the causative agent, which was of a highly infective nature, was conducted.

Methods: Throat swabs were carried out and a multiplex reverse transcriptase-polymerase chain reaction (RT-PCR) assay was performed to identify possible causative factors.

Results: The mean age of patients who had febrile illness during the study period was 20.24 years. The multiplex RT-PCR assay identified respiratory syncytial virus (RSV) as the causative agent. The main symptoms were sore throat $(76.0 \%)$, sputum (72.8\%), cough (72.1\%), tonsillar hypertrophy (67.9\%), and rhinorrhea (55.9\%). The mean temperature was $38.75^{\circ} \mathrm{C}$ and the attack rate among the recruits was $15.7 \%$ (588 out of 3750 recruits), while the mean duration of fever was 2.3 days. The prognosis was generally favorable with supportive care but recurrent fever occurred in $10.1 \%$ of the patients within a month.

Conclusions: This is the first epidemiological study of an RSV outbreak that developed in a healthy young adult group. In the event of an outbreak of an acute febrile illness of a highly infective nature in facilities used by a young adult group, RSV should be considered among the possible causative agents.

Key words: Disease outbreaks, Fever, Military facilities, Military personnel, Respiratory syncytial virus infections

\section{INTRODUCTION}

Respiratory syncytial virus (RSV) belongs to the family of Paramyxoviridae and is classified in the genus Pneumovirus. RSV is the most common cause of fatal acute respiratory tract infection

Received: August 20, 2014 Accepted: November 7, 2014

Corresponding author: Jai-Dong Moon, MD, PhD

322 Seoyang-ro, Hwasun 519-763, Korea

Tel: +82-61-379-7788, Fax: +82-61-379-7791

E-mail: jdmoon@chol.com

This is an Open Access article distributed under the terms of the Creative Commons Attribution Non-Commercial License (http://creativecommons.org/licenses/bync/3.0/) which permits unrestricted non-commercial use, distribution, and reproduction in any medium, provided the original work is properly cited. among infants and young children [1]. Virtually every infant has been afflicted with an RSV infection by the age of 4 years, and reinfection may occur throughout life [2,3]. Moreover, RSV infection has been encountered by almost all people in South Korea before reaching adulthood [4]. Mortality data from several studies suggest that RSV is a significant cause of death in early childhood. Such child death comes from an acute lower respiratory infection, especially following pneumococcal pneumonia or Haemophilus influenza type $b[5,6]$. Recent studies indicate that RSV is an important cause of respiratory infection in elderly patients, either those with compromised immunity or inflicted with chronic illness, as well as in adult populations in a special environment, such as military personnel [7-11]. RSV tends to be more variable and clinical manifestations seem less distinctive 
in adults than they are in children, and the viral cause of the infection is often unsuspected [12-15].

Acute febrile respiratory illness occurred collectively in a group of patients in a boot camp of the Republic of Korea Air Force (ROKAF) from May 2011 to July 2011. We conducted an epidemiological investigation of the causative agent, which was of a highly infective nature. This study investigates the significance and clinical features of RSV in respiratory infections in a healthy young adult group. This study intends to describe the clinical course of this infection and to suggest strategies for treatment and prevention in cases of an RSV outbreak.

\section{METHODS}

\section{Case Definition}

A case patient was a person, among military recruits in this boot camp, who was admitted to the medical care center in the boot camp with chief complaints of fever and symptoms of upper respiratory tract illness after May 26, 2011. The hospitalization criteria include cases with an objectively confirmed fever greater than $37.3^{\circ} \mathrm{C}$ in the morning or greater than $37.8^{\circ} \mathrm{C}$ in the afternoon, in addition to cases that require hospitalization, according to the opinion of an Air Force physician, for the prevention or treatment of an infection on the basis of the fever management protocol of the ROKAF (Supplemental Figure 1). Duration of fever was defined as the period from the onset of fever to the time of objective fever disappearance, as observed with periodic body temperature measurements. Tympanic temperature was measured using a digital thermometer (Braun ThermoScan Pro 4000; Welch Allyn, San Diego, CA, USA).

\section{Epidemiological Investigation}

The test for finding the causative pathogen was carried out on June 14,2011 , at the time when the number of new patients had peaked. Twenty-two patients were admitted to the hospital with cardinal symptoms of respiratory tract infection. Among them, 5 patients who underwent diagnostic tests were selected through random sampling by utilizing Microsoft Office Excel 2003. The throat swab samples collected from these 5 patients were sent to the Seoul Clinical Laboratories and Seoul Medical Science Institute for analysis. Throat swab was carried out by smearing the posterior aspect of the pharynx with a sterile cotton swab while the tongue was pressed down with a tongue depressor. The smear was done by rotating the cotton swab $360^{\circ}$, three to four times. Viral transport media were utilized to transport the samples in a refrigerated condition. A multiplex reverse transcriptase-polymerase chain reaction (RT-PCR) assay was performed with a Seeplex RV6 detection kit (Seegene Inc., Seoul, Korea) for six types of respiratory viruses (RSV, adenovirus, influenza $A$, influenza $B$, para-influenza, and metapneumovirus). The PCR amplification was performed using a T3000 thermocycler (Biometra, Göttingen, Germany). A retrospective investigation for all 588 patients was conducted using computerrecorded patient charts. The clinical characteristics of the disease and types of therapeutic regimens were obtained from these records. This study also examined cases with upper respiratory infection (URI) that occurred in the basic training camp of the ROKAF for the last three years. This investigation also examined all the URI-associated diseases in the military. The numbers of patients with diseases recorded as acute nasopharyngitis, acute laryngopharyngitis, acute tonsillitis, tonsillitis, acute URI, or URI were acquired from the system of military medical information management.

\section{Statistical Analysis}

Subjects were classified into four groups, depending on the type of hospital treatment they received: supportive care group, antibiotic group, antiviral group, and antibiotic and antiviral group. The Kruskal-Wallis test and the analysis of covariance (ANCOVA) were used to find differences in body temperature and duration of fever among the groups. The SPSS version 15.0 (SPSS Inc., Chicago, IL, USA) was used for data analysis.

\section{Ethics Statement}

The subjects' medical records were obtained by referencing the military medical information system. This study was approved by the Medical Research Institutional Review Board of the Ministry of National Defense (no. AFMC-12-IRB-014).

\section{RESULTS}

\section{Outbreak Description}

Subjects in this study included new recruits in training at the boot camp of the ROKAF. All ROKAF recruits are trained at this camp. Together, the recruits have used the same dining and training areas for about two months, and two of their quarters are located $100 \mathrm{~m}$ away. Contact with outsiders is prohibited during the training period (Figure 1). Acute febrile illness occurred collectively among military recruits starting from May 26, 2011. On a suspicion of influenza A (H1N1) in the early phase of 
an epidemic, a rapid antigen test, an immunofluorescence assay, and a multiplex RT-PCR assay were carried out for all 11 patients with early onset of fever at the Armed Forces Medical Research Institute, South Korea, on June 3, 2011. However, these results were all negative. Calling attention to thorough patient examination, segregation, and preventative activities to prevent further incidences did not reduce the incidence of febrile disease, owing to its clustered occurrence since June 9, 2011. Preventative measures included the management of personal hygiene, such as the wearing of a mask by all recruits, thorough hand washing, and restricted sharing of personal items. Further measures comprised instantaneous reporting of symptom development, medical diagnosis, treatment, and segregation. Additional preventative measures for food-associated personnel were reinforced. Patients largely had complaints of fever of $38^{\circ} \mathrm{C}$ or higher, as well as other URI symptoms. Their symptoms were managed well by administration of antipyretics and antibiotics, as well as supportive care, such as fluid therapy. Patients with such symptoms improved after about one to two days of hospital treatment and were discharged from the hospital. The incidence of acute febrile disease continued for approximately two months, but it ceased after July 27, 2011, which marked the end of the epidemic.

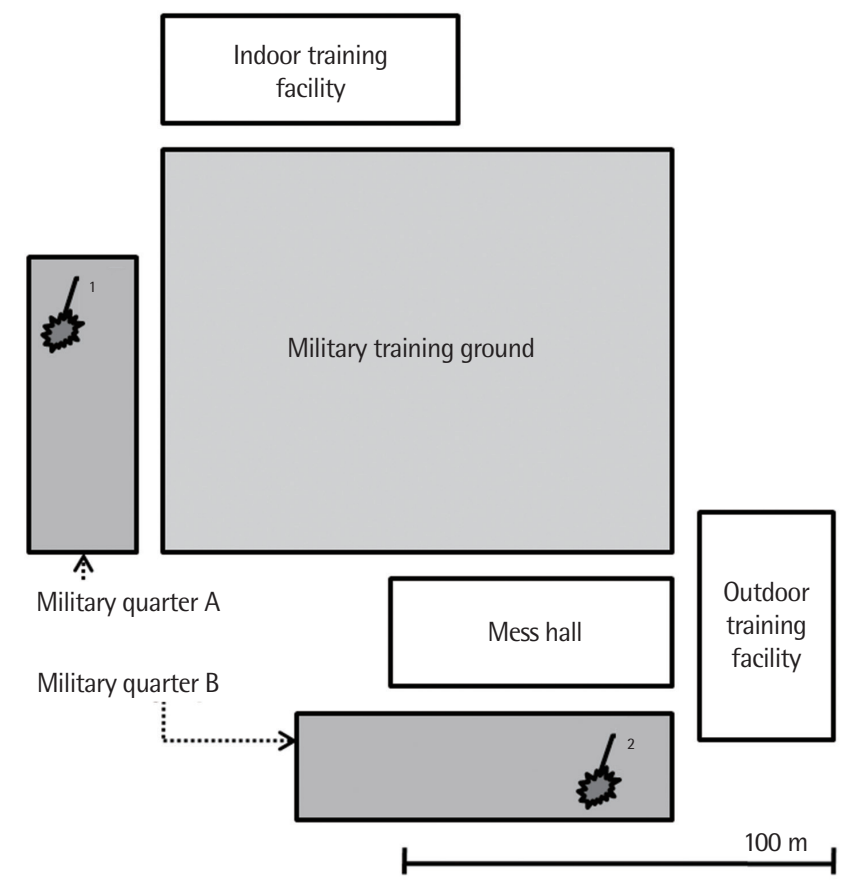

Figure 1. Training camp facilities and possible sites of transmission of respiratory syncytial virus infection. 'Suspected index case in respiratory syncytial virus outbreak, May 26, 2011. ${ }^{2}$ Suspected first case in military quarter B, June 1, 2011.

\section{Attack Rate}

Training for newly arrived recruits continued at this boot camp throughout the year. Of the total of 3750 newly arrived recruits at this camp, 588 developed acute febrile illness, resulting in an attack rate of $15.7 \%$ during this period.

\section{Epidemic Curve}

The epidemic curve depicts the number of URI patients who were cared for at the outpatient department of this boot camp and the number of patients who were admitted to this hospital during the three-year period from 2010 to 2012 (Figure 2). The graph demonstrates that the number of URI patients in the period from May to July 2011 was higher than that in an ordinary year. Any clustered occurrence of acute febrile illness had to be reported to the central epidemiological investigation team and was subjected to the fever management protocol. In times of clustered occurrences of acute febrile illness, as opposed to ordinary years when there is less development of acute febrile illness, as a matter of duty, such incidences must be reported to the Medical Division of Headquarters, which should be followed up by an epidemiological investigation in accordance with the Fever Management Protocol. Until the present (April $11,2014)$, there have been only two epidemiological investi-

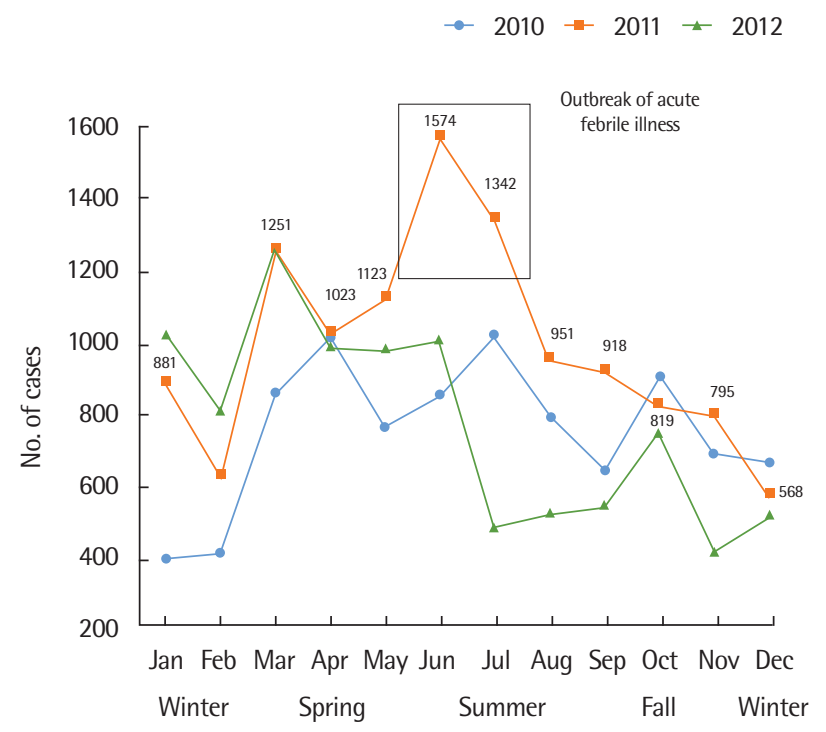

Figure 2. The number of patients with an acute upper respiratory infection who visited the medical care center in the basic training camp of the Republic of Korea Air Force each year (2010 to 2012). The number of recruits in this camp is almost the same each year. 
gations since the protocol was carried out in 2008: H1N1 in 2009 and the outbreak referred to in this study. The occurrence of patients with acute febrile illness continued for 63 days, showing a propagated epidemic curve (Figure 3). The peak occurrence was observed on June 16,2011 , when as many as 30 new patients were admitted to the hospital with a chief complaint of fever.

\section{Laboratory Results}

A multiplex RT-PCR assay was carried out for six types of respiratory viruses (RSV, adenovirus, influenza $A$, influenza $B$, parainfluenza, and metapneumovirus). The assay result was RSV-positive for all 5 subjects, and it was negative for all other viruses.

\section{IIIness Characteristics}

Of the total of 588 case patients, 408 for whom the chart record had not been omitted were investigated. Among them, 280 patients $(68.6 \%)$ had a fever of $38.5^{\circ} \mathrm{C}$ or higher. Their main symptoms were consistent with those of URI, such as sore throat, sputum, cough, tonsillar hypertrophy, rhinorrhea, chill, and/or pharyngeal injection (Table 1). Their mean age was 20.24 years. These patients had a fever, with a mean body temperature of $38.75^{\circ} \mathrm{C}$, and the mean duration of fever was 2.29 days. About $10.1 \%$ of those patients who had been discharged from the hospital developed a recurrent fever within a month and were treated again. They had a fever with a temperature lower than that of

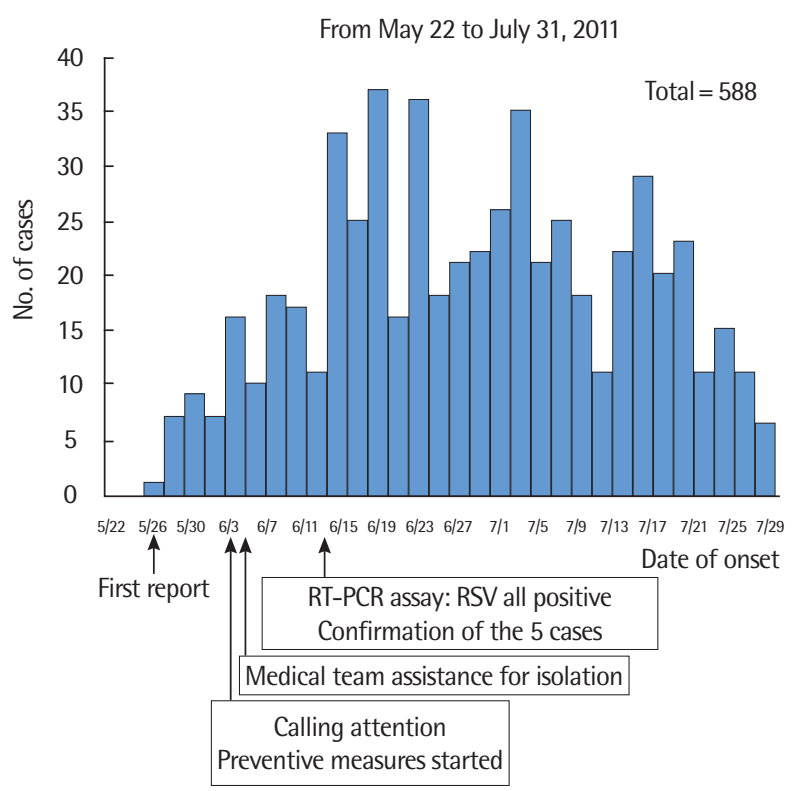

Figure 3. The epidemic curve: the distribution of 588 febrile cases by date of symptom onset. RT-PCR, reverse transcriptasepolymerase chain reaction; RSV, respiratory syncytial virus. the first hospital admission, and a shorter fever duration, showing a satisfactory clinical course. The result of chest $X$-rays showed that 4 patients had pneumonia, but supportive care for all of these patients proved to be effective within a week and they were discharged from the hospital (Table 2).

This study was conducted to find any difference in the duration of fever depending on the type of treatment method. The selection of treatment method was made by Air Force physicians, without consultation with regulatory guidelines. The group that received only supportive care included 14 patients and had a mean fever duration of 1.57 (standard deviation [SD], 1.45) days, showing the fastest recovery. Six patients who received antiviral treatment (oseltamivir) under a suspicion of

Table 1. Symptoms reported by febrile patients $(n=408)$

\begin{tabular}{lc}
\hline Symptoms & $\mathbf{n}(\%)$ \\
\hline Temperature $\geq 38.5^{\circ} \mathrm{C}$ & $280(68.6)$ \\
Sore throat & $310(76.0)$ \\
Sputum & $297(72.8)$ \\
Cough & $294(72.1)$ \\
Tonsillar hypertrophy & $277(67.9)$ \\
Rhinorrhea & $228(55.9)$ \\
Chills & $201(49.3)$ \\
Pharyngeal injection & $132(32.4)$ \\
Headache & $119(29.2)$ \\
Myalgia & $88(21.6)$ \\
General weakness & $31(7.6)$ \\
Vomiting & $10(2.5)$ \\
Diarrhea & $9(2.2)$ \\
Dyspepsia & $6(1.5)$ \\
Abdominal pain & $4(1.0)$ \\
\hline
\end{tabular}

Table 2. Clinical characteristics of febrile patients

\begin{tabular}{lc}
\hline Characteristics & Study population $(\mathbf{n}=\mathbf{4 0 8})$ \\
\hline Age $(y)$ & $20.24 \pm 1.26(18,27)$ \\
Temperature $\left({ }^{\circ} \mathrm{C}\right)$ & $38.8(37.0,40.3)$ \\
Fever duration $(\mathrm{d})$ & $2.29 \pm 1.43(1,8)$ \\
Recurrent febrile illness & $44(10.1)$ \\
Recurrent period (d) & $15.9 \pm 7.42(3,30)$ \\
Temperature $\left({ }^{\circ} \mathrm{C}\right)$ & $38.1(37.1,40.1)$ \\
Fever duration (d) & $1.76 \pm 0.70(1,3)$ \\
Chest radiographic findings & \\
X-ray performed & $91(22.3)$ \\
X-ray hyperinfiltration & $14(3.4)$ \\
Pneumonia diagnosis & $4(1.0)$ \\
\hline
\end{tabular}

Values are presented as mean \pm standard deviation (range), median (range), or $n(\%)$. 
$\mathrm{H} 1 \mathrm{~N} 1$, had the longest mean fever duration of 4.00 (SD, 1.55) days. 348 patients who received antibiotic therapy had a mean fever duration of 2.11 (SD, 1.21) days. Forty patients, who were administered concurrently with antibiotic therapy and an antiviral regimen (oseltamivir) had a mean fever duration of 3.85 (SD, 1.93) days. There was no difference in body temperature among patients receiving the various treatment modalities $(p=0.224$, Kruskal-Wallis test), but a significant difference was found in fever duration among the patients $(p<0.001)$. After making adjustments for the effect of body temperature and number of symptoms, the difference was still significant ( $p<0.001$, ANCOVA).

\section{DISCUSSION}

In the South Korea, hundreds of thousands of healthy young men in their early twenties join the military every year, by entering boot camps. These young recruits are confined to the barracks and restricted from contacting outsiders during the basic military training period. The compromised immune capacity, due to the dense population and high physical and psychological stresses in the boot camp, leads to numerous opportunities for respiratory infection. Residential crowding is known to be a risk factor for RSV infections [16]. The results of studies up to now have revealed that the causative agents of military respiratory infectious diseases are adenoviruses, influenza A and B viruses, Streptococcus pneumoniae, Streptococcus pyogenes, Chlamydia pneumoniae, Mycoplasma pneumoniae, Epstein-Barr virus, coronavirus, and rhinoviruses [17].

A multiplex RT-PCR assay identified RSV as the causative agent in this study. The mean age of the febrile patients was 20.24 years. The main symptoms were sore throat $(76.0 \%)$, sputum $(72.8 \%)$, cough $(72.1 \%)$, tonsillar hypertrophy $(67.9 \%)$ and rhinorrhea (55.9\%). The mean body temperature was $38.75^{\circ} \mathrm{C}$ and the group's attack rate was $15.7 \%$ (588 out of 3750 recruits), while the mean duration of fever was 2.3 days. The prognosis was generally favorable with supportive care. The results of this study support existing data that not only infants but also healthy adults are readily infected, and that the disease in a healthy young adult group tends to be milder and the individuals are able to fight off the virus with relative ease [1822]. The recurrence rate within a month is about $10 \%$. Accordingly, adequate patient explanation and education to encourage the patient to visit the department of medical service as rapidly as possible in recurrent cases of fever could prevent potential additional infections.

The significance of this study is that it is the first investigation of an RSV outbreak that developed in a healthy young adult group. This epidemiological investigation suggests the prospect of RSV as a causative pathogen of respiratory tract infections that could intensively occur among young recruits undergoing basic military training. The RSV-caused URI would not foster immunity in patients, and re-infection might develop throughout one's life. This epidemic with RSV as the etiologic agent had the characteristic manifestations of a common cold, such as high fever and infectivity. Supportive care in the acute phase for two to three days led to a favorable prognosis. None of the patients had any severe complications, and all of them returned to the barracks after the treatment and safely completed their training programme. Nevertheless, this epidemic, which lasted for two months, led to a setback in the military timetable and might have caused damage to military strategy.

Massive antibiotic utilization in the military may increase antibiotic resistance. Fever duration was significantly longer in the group of patients administered an antibiotic or antiviral drug (oseltamivir), despite the fact that there had been no difference in body temperature measured after their hospital admission. Such results may be due to the fact that patients with severe symptoms and long-term hospitalization received an additional antibiotic or antiviral agent. Moreover, patients who delayed seeking care might be more likely to receive an antibiotic or antiviral agent. Another explanation for such results would be the fact that the antibiotic treatment or inappropriate antiviral regimen might not have been particularly effective in the RSV epidemic. This would provide the basis for a suggestion that favorable prognosis in RSV infections might be attained by supportive care in a healthy young adult group. This study verified that there could be a possibility of reoccurrences of RSV-induced epidemics, and supportive care alone would lead to an amicable prognosis in a healthy young adult group.

The major limitations of this study were the fact that the PCR assay only measured samples from five patients, and also that RSV subtypes have not been investigated. The fact that patients with fever largely had similar clinical manifestations, and that five randomly selected patients, among 22 symptomatic subjects, were all RSV positive, would highly support the idea of RSV as the causative agent of this epidemic. In the test performed after 5 patients had been randomly selected from 22 inpatients, the fact that all 5 patients were positive suggested the probability that many more of the inpatients might have been RSV positive. Utilizing the fever management protocol, the ROKAF has monitored the collective development of acute 
febrile illness since 2008. However, the fact that there were only two outbreaks since that time, H1N1 outbreak in 2009 and the outbreak examined in this investigation, points to the possibility that they were caused by the same pathogen. Furthermore, given that the subjects of this study inhabited their quarters, dining and training areas together for an extended period of time, this epidemiological association may be quite high. The epidemic curve also showed the typical pattern of a droplet-mediated respiratory infection with manifestations of infections inflicted by contacts among people. The symptomatic manifestation of upper respiratory tract infection in adults, unlike that of infants, concurred with that of other previous studies [20-22]. An additional limitation was the fact that some military trainees had refused medical care due to concern for their training scores. According to the fever management protocol, it is obligatory that any military trainee with a fever must receive medical treatment. In consideration of this aspect, the actual attack rate could be higher, and this might explain the cause for the extended epidemic lasting as long as two months. The introduction of an infrared thermal camera system may be necessary to promptly identify patients with a fever in the military boot camp. Of the 588 cases, only 408 patients with sufficient chart records were analyzed. This may have led to the exclusion of mild cases and a depiction of the outbreak as more severe than it was. This consideration may, however, support the result of this study that healthy young adults with an RSV infection might have a good prognosis even when receiving only simple supportive care.

In an attempt to prevent a military RSV epidemic, the introduction of an effective RSV vaccine, for which studies are currently in progress, would be worth consideration, if it could be made commercially available [23]. In the event of an epidemic of acute febrile respiratory illness of a highly infective nature, it is recommended to conduct a test for RSV in the young adult population in the military facilities. Clinical courses can be predicted through such activity, and they may also decrease unnecessary antibiotic use. Clinicians may also consider an appropriate antiviral regimen, such as ribavirin, when required [24-26]. The strong possibility that RSV was the causative pathogen for the collective development of this acute febrile illness in the military services foretells that incidences of such infection may multiply rapidly for a long time, as witnessed in this epidemiologic investigation. Thus, rapid detection and segregation of febrile patients, along with installation of an infrared thermal camera system, are necessary. Furthermore, personal hygiene should be promoted. RSV is unstable in the environment, surviving only a few hours on environmental surfaces, and it is readily inactivated with soap and water [18]. Methods of preventing RSV infection include not sharing utensils or cups, avoiding kissing others, covering coughs and sneeze, and washing hands frequently and correctly [19]. And military movement should only be considered when patients are totally recovered and without infectivity. Owing to the characteristics of frequent military mobility, there is a high tendency for propagation of respiratory viral infections to personnel in other military units. In an attempt to prevent those military trainees with high infectivity from moving to other military units during this epidemic, they were directed to move to other military facilities after they had been completely recovered and their infectivity lost. Such measures could have prevented the further spread of this epidemic to other military units. Military planners must focus on how best to control the spread of infectious respiratory diseases in highly mobile military populations [27].

\section{ACKNOWLEDGEMENTS}

This research was supported by the Korean Military Medical Research Project funded by the ROK Ministry of National Defense (ROK-MND-2012-KMMRP-010).

\section{CONFLICT OF INTEREST}

The authors have no conflicts of interest with the material presented in this paper.

\section{REFERENCES}

1. Murray PR, Rosenthal KS, Kobayashi GS, Pfaller MA. Medical microvirology. 4th ed. St. Louis: Mosby; 2002, p. 532-533.

2. Parveen S, Broor S, Kapoor SK, Fowler K, Sullender WM. Genetic diversity among respiratory syncytial viruses that have caused repeated infections in children from rural India. J Med Virol 2006;78(5):659-665.

3. Hall CB, Weinberg GA, Iwane MK, Blumkin AK, Edwards KM, Staat MA, et al. The burden of respiratory syncytial virus infection in young children. N Engl J Med 2009;360(6):588-598.

4. Park WJ. Seroprevalence of respiratory syncytial virus $\lg G$ among healthy young adults in basic training for the Republic of Korea Air Force. J Korean Med Sci 2014;29(9):1325-1327.

5. Thompson WW, Shay DK, Weintraub E, Brammer L, Cox N, Anderson $\mathrm{LJ}$, et al. Mortality associated with influenza and respi- 
ratory syncytial virus in the United States. JAMA 2003;289(2): 179-186.

6. Nair H, Nokes DJ, Gessner BD, Dherani M, Madhi SA, Singleton $\mathrm{RJ}$, et al. Global burden of acute lower respiratory infections due to respiratory syncytial virus in young children: a systematic review and meta-analysis. Lancet 2010;375(9725):1545-1555.

7. Dowell SF, Anderson LJ, Gary HE Jr, Erdman DD, Plouffe JF, File $\mathrm{TM} \mathrm{Jr}$, et al. Respiratory syncytial virus is an important cause of community-acquired lower respiratory infection among hospitalized adults. J Infect Dis 1996;174(3):456-462.

8. O'Shea MK, Ryan MA, Hawksworth AW, Alsip BJ, Gray GC. Symptomatic respiratory syncytial virus infection in previously healthy young adults living in a crowded military environment. Clin Infect Dis 2005;41(3):311-317.

9. O'Shea MK, Pipkin C, Cane PA, Gray GC. Respiratory syncytial virus: an important cause of acute respiratory illness among young adults undergoing military training. Influenza Other Respir Viruses 2007;1(5-6):193-197.

10. Falsey AR, Formica MA, Hennessey PA, Criddle MM, Sullender WM, Walsh EE. Detection of respiratory syncytial virus in adults with chronic obstructive pulmonary disease. Am J Respir Crit Care Med 2006;173(6):639-643.

11. Terrosi C, Di Genova G, Martorelli B, Valentini M, Cusi MG. Humoral immunity to respiratory syncytial virus in young and elderly adults. Epidemiol Infect 2009;137(12):1684-1686.

12. Murata Y, Falsey AR. RSV infection in elderly adults. In: Cane P, editor. Respiratory syncytial virus. Vol. 14. Amsterdam: Elsevier; 2007, p. 163-176.

13. Walsh EE, Falsey AR. Respiratory syncytial virus infection in adult populations. Infect Disord Drug Targets 2012;12(2):98-102.

14. Hall CB. Respiratory syncytial virus and parainfluenza virus. $\mathrm{N}$ Engl J Med 2001;344(25):1917-1928.

15. Kim CK, Choi J, Callaway Z, Kim HB, Chung JY, Koh YY, et al. Clinical and epidemiological comparison of human metapneumovirus and respiratory syncytial virus in seoul, Korea, 2003-2008. J Korean Med Sci 2010;25(3):342-347.

16. Colosia AD, Masaquel A, Hall CB, Barrett AM, Mahadevia PJ, Yogev R. Residential crowding and severe respiratory syncytial virus disease among infants and young children: a systematic literature review. BMC Infect Dis 2012;12:95.

17. Gray GC, Callahan JD, Hawksworth AW, Fisher CA, Gaydos JC. Respiratory diseases among U.S. military personnel: countering emerging threats. Emerg Infect Dis 1999;5(3):379-385.

18. Fraire $A E$, Woda BA, Welsh RM, Kradin RL. Viruses and the lung: infections and non-infectious viral-linked lung disorders. Heidelberg: Springer; 2014, p. 95-99.

19. Centers for Disease Control and Prevention. Brief report: respiratory syncytial virus infection. Frequently asked questions [cited 2014 Oct 8]. Available from: http://www.cdc.gov/rsv/about/faq. html.

20. Agius G, Dindinaud G, Biggar RJ, Peyre R, Vaillant V, Ranger S, et al. An epidemic of respiratory syncytial virus in elderly people: clinical and serological findings. J Med Virol 1990;30(2): 117-127.

21. Hall CB, Long CE, Schnabel KC. Respiratory syncytial virus infections in previously healthy working adults. Clin Infect Dis 2001; 33(6):792-796.

22. Xiang Z, Gonzalez R, Ren L, Xiao Y, Chen L, Zhang J, et al. Prevalence and clinical characteristics of human respiratory syncytial virus in Chinese adults with acute respiratory tract infection. J Med Virol 2013;85(2):348-353.

23. Hurwitz JL. Respiratory syncytial virus vaccine development. Expert Rev Vaccines 2011;10(10):1415-1433.

24. Krilov LR. Respiratory syncytial virus disease: update on treatment and prevention. Expert Rev Anti Infect Ther 2011;9(1): 27-32.

25. Wright M, Piedimonte $G$. Respiratory syncytial virus prevention and therapy: past, present, and future. Pediatr Pulmonol 2011;46(4):324-347.

26. Hynicka LM, Ensor CR. Prophylaxis and treatment of respiratory syncytial virus in adult immunocompromised patients. Ann Pharmacother 2012;46(4):558-566.

27. Trei JS, Johns NM, Garner JL, Noel LB, Ortman BV, Ensz KL, et al. Spread of adenovirus to geographically dispersed military installations, May-October 2007. Emerg Infect Dis 2010;16(5): 769-775. 


\section{Journal of}

Preventive Medicine

\& Public Health

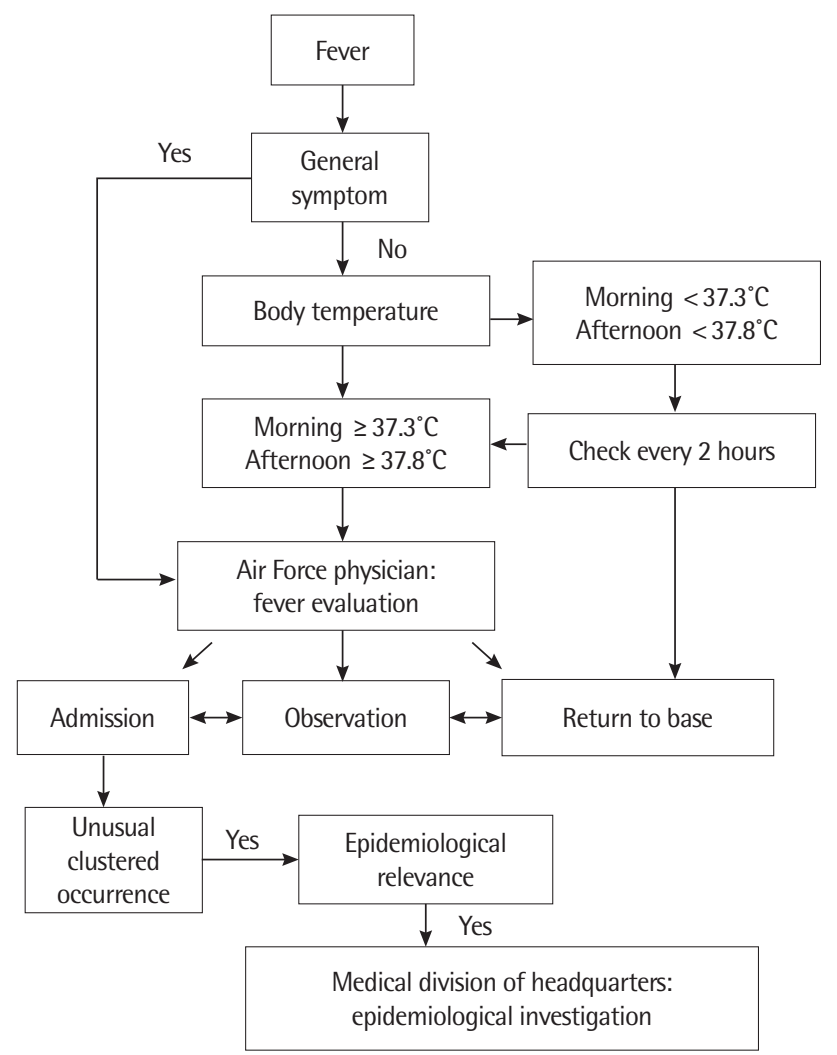

Supplemental Figure 1. Fever management protocol of the Republic of Korea Air Force. 